\title{
GOBERNANZA DELIBERATIVA EN LA GESTIÓN DE CUENCAS HIDROGRÁFICAS: ANALIZANDO LAS CONSECUENCIAS DE LA DIRECTIVA MARCO DEL AGUA EN CATALUÑA
}

\author{
Marc Parés ${ }^{1}$, Alba Ballester ${ }^{2}$, Josep Espluga y Quim Brugué \\ Departamento de Geografía e Instituto de Gobierno y Políticas Públicas. Universidad Autónoma de Barcelona ${ }^{1}$ \\ Instituto de Gobierno y Políticas Públicas. Universidad Autónoma de Barcelona²
}

\section{RESUMEN}

Los primeros planes de cuenca previstos en la Directiva Marco del Agua fueron aprobados en España muy recientemente, motivo por el cual todavía no disponemos de estudios que evalúen los resultados de los procesos participativos llevados a cabo. En este artículo se analiza una experiencia participativa que, por su carácter ejemplar y atípico, ha sido considerada una buena práctica: el proceso deliberativo del Distrito de Cuenca Fluvial de Cataluña. Nos interrogamos sobre las consecuencias de la deliberación y, más concretamente, sobre si un buen proceso de deliberación mejora la política de aguas. Es decir, investigamos la relación entre las características procedimentales de la deliberación y sus resultados. Para ello combinamos datos cuantitativos con información cualitativa obtenida a partir de análisis documental y treinta entrevistas en profundidad. Las limitaciones de la estrategia comunicativa, la poca integralidad con la que se planifica la política de aguas y la falta de una cultura política deliberativa son algunas de las debilidades identificadas en el caso estudiado. Concluimos que un buen proceso deliberativo es una condición necesaria pero no suficiente para garantizar un impacto significativo sobre la política de aguas.

Palabras clave: Directiva Marco del Agua, gobernanza, participación, democracia deliberativa

\section{ABSTRACT}

\section{Deliberative governance on river basin management planning: analysing the consequences of the Water Framework Directive in Catalonia.}

The first river basin management plans under the Water Framework Directive have been adopted in Spain recently. For thus, there are no studies evaluating the results of the participatory processes carried out. This article discusses a participatory experience that has been considered a good practice: the deliberative process of River Basin Management planning in Catalonia. We are interested on the consequences of deliberation and, more specifically, we want to know if a good deliberative process improves water policy. Drawing upon quantitative and qualitative data obtained from documentary analysis and thirty interviews, we investigate the relationship between the procedural features of deliberation and its results. The limitations of the communication strategy, the lack of comprehensiveness with which water policy planning has been made and the lack of a deliberative political culture are some of the weaknesses identified in the case study. We conclude that a good deliberative process is a necessary but not a sufficient requierement to ensure a significant impact on water policy.

Keywords: Water Framework Directive, governance, participation, deliberative democracy

\section{INTRODUCCIÓN}

Con el fin de lograr un buen estado ecológico de todas las masas de agua europeas en 2015, la Directiva Marco del Agua (DMA en adelante) promueve la participación activa de las partes interesadas

\footnotetext{
Contacto: Marc Parés: marc.pares@uab.cat; Alba Ballester: alba.ballester@gmail.com; Josep Espluga: joseplluis.espluga@uab.cat; Quim Brugué: quim.brugue@uab.cat
} 
y el público en general en las políticas de aguas. Más concretamente, en su artículo 14 la DMA establece que el éxito de la misma depende de "una colaboración estrecha y una actuación coherente de la Comunidad, los Estados miembros y las autoridades locales, así como de la información, las consultas y la participación del público, incluidos los usuarios". Al mismo tiempo, la directiva establece que, en particular, la participación de las partes interesadas debe llevarse a cabo en la elaboración, revisión y actualización de los planes de gestión de cuencas hidrográficas.

La DMA, pues, apuesta por lo que podemos llamar un modelo de gobernanza participativa a partir de unos objetivos sustantivos pre-establecidos (Sabel y Zeitlin, 2008). Sin embargo, a partir de estas directrices son los Estados miembros y las unidades político-administrativas inferiores los que tienen libertad para avanzar en estos objetivos como mejor les parezca. La Directiva, por lo tanto, no es una legislación estricta, sino que los Estados miembros pueden interpretarla de diferentes maneras (Kallis y Butler, 2001; Lanz y Scheuer, 2001). Tal y como ya apuntó Kaika (2003), la Directiva otorga un papel central a los Estados nacionales en la fase de implementación de la misma, pues son ellos (o sus regiones) quienes deben establecer cómo se articulan esas redes de gobernanza, con qué actores, con qué métodos y con qué finalidad. De acuerdo con esto, la DMA se ha implementado de manera muy diferente en cada Estado miembro y sus regiones. Como resultado observamos que, una vez finalizada la fase de elaboración de los planes de cuenca, los procesos participativos llevados a cabo en los distintos lugares responden a objetivos y métodos muy variados. Eso no debería ser un problema, pues parece lógico que las formas de participación se adecuen a las características socioambientales de cada realidad territorial. Lo que ocurre, sin embargo, es que las diferencias entre cuencas no son únicamente relativas al formato que toma la participación sino, sobretodo, muestran distintos grados de participación de los ciudadanos, con distinta capacidad de incidencia sobre los resultados finales. Aunque se han realizado varios estudios que discuten el papel y los beneficios de la participación pública en la aplicación de la DMA (Mostert, 2003; Newig et al, 2005), analizan el cambio que ello supone en la política de aguas (Huitema y Meijerink, 2010 ) y sistematizan los pros y los contras de los diferentes métodos de participación (Antunes et al, 2009; De Stefano, 2010; Kallis, 2006), existe poca investigación que se aproxime al análisis de la gobernanza participativa en la DMA a partir del estudio de las características estructurales de las redes participativas en su conjunto, de forma integral y más allá del propio proceso participativo (Newig et al., 2010). En este artículo adoptamos este último enfoque.

Durante la última década, las investigaciones han mostrado un creciente interés por la implementación participativa de la DMA. Hasta la fecha, sin embargo, han tendido a centrarse en el análisis procedimental de la participación, prestando muy poca atención a los efectos que la participación ciudadana ha tenido en la política de agua. Tal hecho podría explicarse porque, aunque los planes de cuenca previstos en la DMA deberían haber sido aprobados en 2009, en muchos países el proceso ha tenido un retraso significativo. Es decir, no era posible analizar los efectos de la participación simplemente porque los planes de cuenca no habían sido finalizados. En España, por ejemplo, Espluga et al. (2011) analizaron en profundidad los procesos participativos llevados a cabo. Sin embargo, la evaluación no incluyó los efectos que estos procesos han tenido en los planes de cuenca debido a que estos planes aún no se habían aprobado cuando se realizó dicha investigación. Con el objetivo de llenar este vacío, el propósito de este trabajo es examinar las consecuencias de un proceso deliberativo concreto, el del Distrito de Cuenca Fluvial de Cataluña, cuyo plan de medidas fue aprobado por el gobierno catalán a finales del año 2010.

El debate sobre las relaciones entre los procedimientos y los efectos de la participación no es una cuestión exclusiva de la DMA. La mayor parte de la literatura general sobre la teoría deliberativa ha girado alrededor del ideal normativo de la democracia deliberativa en relación a sus procedimientos, las condiciones en las que se produce la deliberación y los objetivos de la misma (Bohman y Rehg, 1997). En este contexto, una de las discusiones más significativas entre los teóricos deliberativos es la que se produce entre los procedimentalistas y sustantivistas (Gutmann y Thompson, 2004). Mientras que el procedimentalismo puro sostiene que los principios deliberativos deben aplicarse sólo al proceso de formulación de las políticas públicas, los partidarios de una concepción más sustantiva niegan que los principios procesales sean suficientes para lograr una democracia deliberativa, ya que estos pueden producir resultados injustos. La mayoría de los estudios recientes sobre la gobernanza deliberativa han puesto su atención en cuestiones procedimentales como la inclusión de todas las voces, el respeto mutuo 
entre los oponentes, la economía del desacuerdo moral o el cambio en las posiciones de entrada (Bobbio, 2010; Hendriks, 2006; Sunstain y Hastie, 2008). Menos estudios, sin embargo, han explorado los efectos (los resultados) de deliberación (Hendriks et al., 2007; Lo et al., 2013; Schkade et al., 2006).

Ciertamente, varios estudios empíricos han centrado su atención en cómo la participación pública mejora (o no) los procesos de formulación de las políticas. Algunas de estas investigaciones, además, se interesan por los procesos participativos vinculades a cuestiones territoriales o ambientales como la ordenación del territorio o la gestión de recursos naturales (Baker et al., 2010; Conrad et al., 2011; Groves et al., 2013; Parés, 2011). Sin embargo, como apuntan Blanco y Lowndes (2011), a pesar del claro aumento de las iniciativas participativas por parte de los gobiernos de distinta índole, hay pocas evidencias relativas a los efectos de la participación.

Más allá de los efectos que podría tener la participación, la mayoría de los estudios han examinado los factores explicativos de los resultados de la participación. Algunos autores han destacado la importancia de la calidad deliberativa como un hecho que determina los resultados de la participación (Parés, 2009; Papadopoulus y Warin, 2007). Fung (2003), por ejemplo, describe diferentes opciones de diseño institucional de la participación y analiza sus consecuencias, argumentando que el diseño de la participación condiciona sus efectos. Por otro lado, Blanco y Ballester (2011), basándose en diferentes aportaciones teóricas, identifican cinco condiciones para mejorar la capacidad de transformación que tiene la participación. Algunas de estas condiciones son cuestiones procedimentales, mientras que otras están relacionadas con el comportamiento de los actores o el contexto en que se desarrolla el proceso participativo. Según estos autores, las cinco condiciones para mejorar los efectos de la participación son: un liderazgo político tanto del proceso participativo como de las políticas afectadas (Haus et al., 2005), un enfoque holístico de la política pública sometida a participación (Wagenaar, 2007), una amplia visibilidad del proceso participativo llevado a cabo (Font y Blanco, 2006), la capacidad de impacto de los ciudadanos en las políticas públicas a través del proceso de participación (Arnstein, 1969; Fung, 2006) y un verdadero cambio cultural de políticos, técnicos y ciudadanos (Subirats, 2003; Brugué, 2004). Sobre la base de esta literatura, nuestro trabajo combina dos dimensiones analíticas (las pautas de funcionamiento y las pautas de comportamiento) con el fin de comprender plenamente las consecuencias de un proceso de deliberación concreto realizado de acuerdo con la DMA.

El estudio de caso que hemos seleccionado para nuestra investigación es la aplicación de la DMA en Cataluña. Hemos seleccionado este estudio de caso porque es un caso ejemplar y en muchos términos también atípico (Ruddin, 2006; Yin, 2003). El gobierno de Cataluña jugó un papel clave en la promoción de un proceso deliberativo de grandes dimensiones en el Distrito Fluvial de Cataluña, gestionado íntegramente por la administración autonómica. La intensidad del proceso de deliberación, la ambición del mismo, los recursos invertidos y el compromiso político del gobierno, nos llevan a concluir que el caso catalán puede ser considerado como una "buena práctica", especialmente si se compara con otras experiencias participativas sobre la gestión del agua en Europa.

El proceso deliberativo de la DMA en Cataluña representa una oportunidad única para hacer frente a dos grandes debates relacionados con la gestión del agua y la gobernanza deliberativa. En primer lugar, como buena práctica, este estudio de caso nos permitirá evaluar empíricamente los efectos de un proceso de deliberación real, analizando los hechos que permiten o restringen el éxito de la deliberación. En segundo lugar, como buena práctica deliberativa aplicada a la gestión del agua, este estudio de caso nos permitirá averiguar si, como se espera de la DMA, un buen proceso de deliberación mejora la política de gestión de las cuencas hidrográficas.

Basándose en la evaluación de este proceso de deliberación y combinando datos cuantitativos y cualitativos, la investigación que aquí se presenta analiza las consecuencias de la deliberación (centrado nuestra atención en las mejoras producidas en las políticas públicas) y explora las causas que explican sus luces y sus sombras. La evaluación muestra que incluso cuando un proceso deliberativo se lleva a cabo bajo parámetros de calidad democrática (en términos generales) y logra unos resultados significativos, la mayoría de los participantes tienden a estar insatisfechos con los resultados. En este artículo, pues, identificamos aquellos factores que explican no sólo el éxito de la experiencia, sino también la insatisfacción de los participantes. En este sentido, nuestra investigación revela una relación significativa 
entre el proceso (la forma en cómo se ha desarrollado el proceso deliberativo) y los efectos (los resultados tangibles e intangibles producidos por el mismo). Aún así, constatamos también que un buen proceso deliberativo es una condición necesaria pero no suficiente para garantizar un impacto significativo sobre la política de aguas.

Para obtener información cualitativa sobre el proceso participativo se realizaron 30 entrevistas semiestructuradas en profundidad a una muestra de actores involucrados directamente en el proceso. Las entrevistas, por tanto, se dirigieron a ciudadanos que habían formado parte de la experiencia, ya fuera como participantes o como promotores institucionales o profesionales. La selección de personas a entrevistar se realizó buscando tanto la diversidad sectorial (representantes de la administración autonómica y local, organizaciones sociales y ambientales, actores del sector económico-empresarial y actores del sector agro-ganadero y forestal) como también la diversidad territorial, entrevistando a personas de distintas subcuencas en las que se llevaron a cabo procesos deliberativos.

\section{LA GOBERNANZA DEL AGUA EN LA DMA}

\subsection{La aplicación de la DMA en España}

Como apuntamos anteriormente, en su artículo 14 la DMA amplía la participación en la gestión del agua a todas las partes interesadas (no únicamente usuarios, aunque incluidos estos) y al público en general. De manera más concreta, la DMA prevé tres niveles de implicación del público: información, consulta y participación activa. La directiva exige que los dos primeros niveles (información y consulta) estén plenamente "asegurados", mientras que el tercero (la participación activa) debe ser "fomentado". Veamos con un poco más de detalle que entendemos por estos tres conceptos (Espluga et al., 2011)

La Información pública: consiste en proporcionar acceso a la información y difundirla activamente a todas las partes interesadas y al público general, lo cual abarca básicamente dos aspectos. Por un lado, el suministro de información suficiente en las distintas fases de la implantación; por otro lado, el acceso a información y documentos de referencia previstos en la directiva. Como indica La Calle (2009), la información difundida debe permitir obtener la información de base a todo aquel público especializado que quiera conocerla.

La Consulta pública: supone una implicación directa del público, ofreciéndole la oportunidad de reaccionar a las propuestas iniciales de la Administración. Se trata de un proceso en el que se deben recoger los comentarios, experiencias, sugerencias, percepciones e ideas de los agentes consultados. Implica poner los documentos a disposición del público para que éste presente sus observaciones por escrito, organizar audiencias públicas o tratar de recabar activamente los comentarios y opiniones del público mediante, por ejemplo, encuestas y entrevistas.

La Participación activa: es el nivel más alto de participación propuesto por la DMA. Implica la celebración de reuniones específicas con partes interesadas y público en general donde se identifiquen problemas colectivamente y se propongan medidas y actuaciones para afrontarlos. Implica que los diferentes sectores de la población están invitados a contribuir activamente al proceso de planificación deliberando sobre problemas y contribuyendo a la búsqueda de soluciones, es decir, influyendo en mayor o menor grado en la toma de decisiones. La participación activa en el proceso de planificación hidrológica debería involucrar no solamente a las partes tradicionalmente más interesadas en la gestión del agua sino a toda la sociedad en su conjunto, a través de foros o grupos de trabajo específicos.

Aunque en España la gestión del agua ha contado históricamente con la participación de los grandes usuarios, la aplicación de la DMA supone, sin duda alguna, una transformación en las formas de gobernanza del agua en España. La implementación de la directiva conlleva la apertura de las redes de gobernanza del agua a nuevos actores (usuarios no económicos, plataformas ecologistas, etc.), permite la participación del público en general en los procesos de planificación hidrológica, implica la creación de nuevas arquitecturas institucionales de gobernanza (o la modificación de las pre-existentes) y provoca la creación de nuevos procedimientos y nuevas relaciones entre los actores involucrados en la planificación y la gestión del agua. 
Aún así, la aplicación de las directrices fijadas por la DMA han dado lugar a múltiples formas de gobernanza participativa en las distintas cuencas del territorio español.

\subsection{Estudio de caso: el Distrito de Cuenca Fluvial de Cataluña}

En nuestro caso de estudio, el Distrito de Cuenca Fluvial de Cataluña, se hizo una clara apuesta por el fomento de la participación activa, estableciendo un particular modelo de gobernanza deliberativa con el fin de elaborar el Plan de Gestión de Cuenca. Como ya hemos comentado, hemos seleccionado este estudio de caso porque es ejemplar y, en muchos términos, también atípico. Se trata de un distrito de cuenca de carácter intracomunitario y gestionado por la administración autonómica. Aunque el distrito no abarca la totalidad del territorio catalán, sino que únicamente incluye las cuencas internas, cabe destacar que estas tienen una superficie de $16.423 \mathrm{~km}^{2}$, representan el 52\% del territorio catalán y contienen el $92 \%$ de su población.

De hecho, el caso destaca porque el gobierno catalán jugó un papel muy destacado promoviendo un vasto proceso deliberativo en la elaboración del plan de cuenca. Durante 4 años el gobierno catalán invirtió $3 \mathrm{M} €$ en el proceso de deliberación, se llevaron a cabo 290 reuniones, que incluyeron a más de 1.700 personas y se generaron casi 1.000 horas de debate. Más de 1.500 propuestas fueron producidas y 964 de estas propuestas fueron introducidas en el documento final del Plan de Gestión del Distrito de Cuenca Fluvial de Catalunya, aprobado por el gobierno en noviembre de 2010.

Según los documentos de la Agencia Catalana del Agua (ACA), los objetivos del proceso participativo eran "informar y escuchar la opinión de los ciudadanos y ciudadanas, así como a los representantes de entidades, administraciones y empresas, para debatir las propuestas sobre la gestión del agua a las diferente cuencas catalanas". Se señalaba también que "la DMA es el marco legal aprobado por el Parlamento Europeo a finales de 2000 que nos insta a generar estos procesos participativos", indicando que "nuestro gran reto es conseguir un buen estado de las masas de agua". Finalmente, se afirma que "el derecho a la participación es un derecho ciudadano, ya que la ciudadanía tiene derecho a tomar parte en las cuestiones públicas y en las decisiones sobre el futuro de su entorno".

Tomando como punto de partida la valoración sobre el estado de las masas de agua y siguiendo las indicaciones de la DMA, la ACA y la Dirección General de Participación Ciudadana diseñaron un proceso participativo estructurado en 5 fases. De entrada se debían preparar los materiales divulgativos y las bases de datos con las referencias de los actores más significativos de cada cuenca (fase preparatoria). En segundo lugar, había que convocar y celebrar sesiones informativas donde establecer el primer contacto con los actores, conocer sus impresiones, resolver sus dudas e invitarlos al proceso (fase informativa). En tercer lugar, se propusieron unos talleres participativos con el doble objetivo de validar el diagnóstico de partida sobre el estado de las masas de agua, y de recoger propuestas para, eventualmente, incorporarlas a los planes de medidas (fase participativa). En cuarto lugar, se confirmaba el compromiso de documentar el proceso, recogiendo las aportaciones y las opiniones que los diversos actores vertieron durante el proceso participativo (fase de elaboración de las conclusiones). Finalmente, también por mandato de la propia DMA, la ACA se comprometía a evaluar las diferentes propuestas y a rendir cuentas, con argumentos, de su inclusión o no en los planes de medidas (fase de retorno).

Como puede observarse, se trata de unas fases que pretenden, al menos idealmente, articular un proceso deliberativo. Además de estas fases, cabe señalar que el proceso de participación en Cataluña se segmentó en 16 unidades territoriales, las cuales no se justificaban estrictamente en términos hídricos, sino que respondían a la voluntad de acercar los debates a los territorios y a las personas que viven en ellos.

\section{EL PROCESO DELIBERATIVO}

El propósito de este apartado es examinar las características del proceso deliberativo llevado a cabo en el Distrito de Cuenca Fluvial de Cataluña, un proceso que ya ha concluido y que dio lugar a la aprobación del respectivo Plan de Gestión de Cuenca. 
Basaremos nuestro estudio procedimental de esta experiencia deliberativa en dos dimensiones analíticas. En primer lugar, focalizaremos nuestra atención en las pautas de funcionamiento del proceso, analizando tanto los parámetros de calidad deliberativa sugeridos por Gutmann y Thompson (2004) como otros factores relativos al diseño, la organización y la gestión del proceso. Por otro lado, analizaremos las pautas de comportamiento de los actores. Entendemos que, tal y como apuntan Brugué y Parés (2012) ambas dimensiones analíticas son clave para poder explicar los efectos de la deliberación.

\subsection{Pautas de funcionamiento}

Para analizar cómo funcionó el proceso, cómo se organizó y cómo se gestionó, nos centraremos en distintos elementos. En primer lugar, analizaremos las cuatro pautas sugeridas por Gutmann y Thompson (2004) en relación a los procesos deliberativos, según los cuales un buen proceso deliberativo debe propiciar el intercambio de argumentos, debe ser accesible y comprensible, debe producir unos resultados concretos y debe realizarse bajo el respeto mutuo. Sin embargo, aunque se logren las características principales de la calidad deliberativa, hay otros elementos de procedimiento que se deben tener en cuenta para comprender la producción de efectos de deliberación. Así, además de los parámetros relativos a la calidad deliberativa de los procesos, en esta sección analizaremos también aquellas características procedimentales relacionadas con las normas y protocolos que rigieron el proceso, la estrategia comunicativa y los participantes en el proceso.

En términos generales, con algunos matices, podríamos afirmar que el proceso de deliberación evaluado aquí se ha llevado a cabo siguiendo los principales patrones de la calidad deliberativa sugeridos por Gutmann y Thompson (2004).

El primer elemento a destacar es que el proceso permitió, facilitó y propició el intercambio de argumentos entre los participantes. Las siguientes citas de las entrevistas realizadas son una clara prueba de ello:

"Todo el mundo que tenía algo que decir, puedo hacerlo, con todas las facilidades" (empresario)

"Me parece que es algo muy bien hecho, me parece que es un proceso modélico y que ha recogido una buena parte de las sensibilidades que había durante el proceso participativo." (miembro de una organización ambientalista)

“(La dinamización) permitía mantener el ritmo (....) se aseguraba un esquema de funcionamiento que nos permitía discutir y avanzar." (miembro de una organización ambientalista)

La duración y la intensidad del proceso (290 reuniones durante más de 3 años y más de 1.000 horas de debate) permitieron el intercambio de opiniones y argumentos. Además, los talleres de deliberación fueron realizados por empresas consultoras que hicieron el trabajo fundamental para hacer efectivo el intercambio de argumentos, a partir del respeto mutuo entre los participantes. El intercambio de razones propiciaría, según la teoría, una moderación de las posiciones más extremas, o usando otros términos, una mayor propensión al equilibrio. El consenso y el acuerdo entre los participantes no fueron, obviamente, absolutos, aunque el proceso generó unas propuestas suficientemente equilibradas como para encontrar un bajo índice de rechazo por parte de la administración responsable. Así, de las 1.529 propuestas recogidas durante el proceso participativo sólo 51 fueron rechazadas (3\%). Este bajo índice de rechazo no es, obviamente, un indicador directo del equilibrio alcanzado en las propuestas, pero sí que nos podría sugerir indirectamente que las propuestas más "atrevidas" han tendido a no pasar el filtro del propio proceso deliberativo.

En segundo lugar, el proceso fue accesible y lo suficientemente comprensible para los ciudadanos y sus representantes. La información producida era enorme y se podía acceder fácilmente a ella a través de internet. Sin embargo, aunque el proceso fue abierto formalmente a todo el mundo, la estrategia comunicativa se dirigió básicamente a las partes interesadas, como representantes, y no al público en general. Las siguientes citas constatan la accesibilidad y la comprensibilidad de la información producida a lo largo del proceso.

"El proyecto era muy bueno, muy bonito, muy comprensible" (agricultor) 
"Para la sociedad civil en general, yo creo que había una información perfecta" (miembro de una organización ambientalista)

En tercer lugar, más de cuatro años después del inicio del proceso, una decisión práctica permitió ver los resultados. El Gobierno regional aprobó el plan de cuenca, una decisión concreta, que incluyó el 63\% de las propuestas formuladas durante el proceso de deliberación. Por otro lado, sin embargo, hay que señalar que el $34 \%$ de las propuestas no obtuvieron una respuesta administrativa, ya que no eran de la competencia del Departamento de Medio Ambiente y Vivienda.

Por último, aunque se partía de una cierta desconfianza entre los distintos actores, el proceso deliberativo buscó y logró trabajar a partir del respeto mutuo entre las partes. Cuando analicemos las pautas de comportamiento de los actores profundizaremos en esta cuestión.

En lo que se refiere a las normas y protocolos que rigen el proceso, encontramos que las reglas eran claras y seguras, y que eran una garantía para el buen desarrollo del proceso. Las entrevistas muestran una valoración positiva de las normas y protocolos como buenas formas para favorecer el debate entre las partes interesadas. Sin embargo, algunos de los entrevistados han señalado que las reglas se han diseñado de arriba hacia abajo y no son lo suficientemente flexibles como para adaptarse a cada territorio y a sus características.

En cuanto a la estrategia comunicativa detectamos que, a pesar de que el proceso fuera accesible y comprensible, existieron algunas carencias importantes en la estrategia comunicativa. Siguiendo las pautas marcadas por la propia DMA, el proceso dispuso de unos objetivos claros y suficientemente orientadores para organizar el debate. Además, el hecho de acabar con algo tan específico como el plan de cuenca contribuyó a hacer comprensibles los objetivos. Sin embargo, el esfuerzo comunicativo que se realizó no fue suficiente para llegar al conjunto de la población. La difusión del proceso se basó en un sitio web muy completo y en la edición de diversos materiales informativos. El número de participantes, como después detallaremos, fue considerable, pero no dejó de ser una especie de élite que agrupaba a las personas especializadas e interesadas en temas hídricos en Cataluña. La estrategia de comunicación se focalizó en estos segmentos, sin posibilidad de usar un lenguaje y unos medios con capacidad para acceder al conjunto de la opinión pública.

Preguntando en nuestras entrevistas sobre los objetivos y los contenidos del proceso de deliberación, las respuestas eran absolutamente contradictorias. Los miembros de la ACA tendieron a responder que se trataba de un proceso de consulta (sin carácter vinculante) en el que los límites estaban claros con el fin de evitar falsas expectativas. Los agentes económicos y las empresas, por su parte, tendían a mantenerse alejados de las lógicas deliberativas y definían el proceso en términos de negociación. Los grupos de interés agrícolas y forestales, por lo general, eran muy explícitos al hablar sobre el contenido del proceso y, en cambio, resultaban más ambiguos cuando hablaban de sus objetivos. Por último, las organizaciones ambientalistas y sociales se dividen entre los que estaban muy satisfechos con el proceso y consideraban que sus objetivos eran claros y los que criticaban la información y los temas planteados por el proceso. Aquí tenemos una muestra de todas estas contradicciones en torno a los objetivos del proceso:

"Muchos de los participantes pensamos que un proceso participativo era un proceso ejecutivo" (empresario)

"Pensamos que teníamos que ir, sencillamente, sin ningún otro prejuicio" (agricultor)

"La pretendida transparencia de la participación no se dio (...) Muchísima información relevante, sobre todo para los casos más conflictivos, no estaba sobre la mesa. No era lo suficientemente transparente y no todo se ha llevado al debate." (miembro de una organización ambientalista)

"Fue un proceso participativo real" (miembro de una organización ambientalista)

Los objetivos y los contenidos del proceso deliberativo de la DMA, por tanto, no fueron comprendidos de la misma forma por todos los participantes. De hecho, la gran mayoría de los participantes no sabían muy bien lo que estaban haciendo allí ni por qué lo hacían. Esto podría explicarse debido a una mala estrategia comunicativa, sin embargo, la falta generalizada de cultura deliberativa también podría explicar este hecho. La cultura administrativa y gubernamental, de ciudadanos, técnicos y políticos está impregnada de racionalidad tecnocrática y, por tanto, la racionalidad deliberativa suele ser difícil de en- 
tender. Tenemos dificultades tanto para aceptar que las decisiones debatidas pueden ser mejores como para entender las características que debería satisfacer un proceso deliberativo. En este desconcierto, los prejuicios se imponen a los conocimientos, de manera que cada uno interpreta la experiencia participativa más por lo que ya opinaba antes y menos por lo que ha podido observar en la práctica concreta. Además, en un magma de percepciones dispares y cruzadas, el proceso deliberativo se ve atrapado en la proliferación de múltiples y a menudo contradictorias expectativas. Las percepciones de los actores, pues, no tienen porqué ser coincidentes ni ser un reflejo fiel de la realidad. A menudo la distorsionan y presentan una imagen irreal, como se ha visto, aunque, son sus percepciones y tienen incidencia en el desarrollo de la experiencia participativa. El desarrollo de una cultura deliberativa, por otro lado, no es sólo una cuestión de recursos y de procedimiento, es algo que requiere tiempo y maduración.

La otra debilidad de la estrategia comunicativa está relacionada con nuestro siguiente objeto de análisis: los participantes involucrados en el proceso. Como la estrategia comunicativa no era lo suficientemente potente, el proceso se redujo a una élite de participantes procedentes de la sociedad civil organizada, los sectores económicos y las instituciones públicas. Por lo tanto, no se logró involucrar al conjunto de la sociedad (todos los "usuarios del agua" y el "público en general") en el proceso deliberativo. De hecho, la propia estrategia comunicativa y el diseño del proceso fueron pensados para promover y gestionar un proceso de deliberación con las partes interesadas, no con el público en general.

A pesar de ser un proceso deliberativo elitista, hay que destacar que el número de participantes (1.634) y de organizaciones (1.085) implicados fue muy alto y su diversidad también, como podemos ver en la Tabla 1. Sin embargo, no todos los participantes fueron a todas las sesiones que deberían haber asistido y la mayoría de ellos participaron de manera esporádica. Sólo los que tenían un fuerte incentivo mantuvieron el interés participativo, lo que favoreció que la deliberación fuera más un debate entre las partes interesadas que un debate entre los puntos de vista alternativos sobre la política de aguas.

Tabla 1. Participantes por sectores

\begin{tabular}{|l|c|c|}
\hline \multicolumn{1}{|c|}{ Perfil } & Número de participantes & $\%$ \\
\hline Administración autonómica y local & 517 & $31,6 \%$ \\
Organizaciones sociales y ambientales & 476 & $29,1 \%$ \\
Actores económicos y empresariales & 290 & $17,8 \%$ \\
Sector agro-ganadero y forestal & 229 & $14,0 \%$ \\
Ciudadanía no organizada & 122 & $7,5 \%$ \\
Total & 1.634 & $100 \%$ \\
\hline
\end{tabular}

Fuente: Agència Catalana de l'Aigua, 2010

Si nos fijamos en la diversidad de los participantes vemos que, además del predominio de participantes organizados por encima de la sociedad civil no organizada, hay también una sobre-representación de dos colectivos: la administración y las organizaciones sociales y ambientales. Cabe destacar la participación de estas últimas organizaciones, pues se trata de unos actores que tradicionalmente no habían sido tenidos en cuenta en la gestión del agua y que, con la nueva gobernanza deliberativa, han pasado a ser los actores más activos y que más han participado en el proceso deliberativo, muy por encima del sector empresarial o del sector agro-forestal. Estos actores han percibido y han utilizado el proceso deliberativo como una oportunidad para hacer oir su voz. En este sentido la DMA, posibilitando la participación de actores tradicionalmente excluidos de la política de aguas, ha alterado las geometrías de poder vinculadas a la toma de decisiones en este ámbito.

Aún así, si nos fijamos en la significatividad de los actores participantes hay que destacar algunas especificidades. En primer lugar, identificamos la ausencia significativa de algunos agentes económicos, sobre todo en algunos de los territorios en los que tienen efectos importantes en las masas de agua. Tal y como corroboraron muchos de los actores institucionales entrevistados, los agentes económicos por lo general tenían otros medios para influir en el proceso de formulación de la política de aguas al margen de los procesos deliberativos. Así, se reconoce que estos actores utilizan sus recursos y su posición de poder privilegiada en la red de la gobernanza para lograr tener influencia en la política sin tener que 
gastar su tiempo participando en estos mecanismos innovadores de deliberación. Veamos algunas de esas afirmaciones:

"Ellos entendieron que se trataba de un foro ecologista y que ya tenían otro camino privilegiado para mantener la relación con el gobierno" (miembro de la ACA)

"Ellos se reúnen directamente con el Conseller, o con el director de la ACA... los procesos con las personas no son sus procesos" (miembro de la ACA)

"El gran industrial ya tiene acceso a los que toman las decisiones en la politica de aguas, por lo que probablemente no apareció allí (en el proceso de deliberación)" (miembro de la administración autonómica)

En definitiva, observamos que, tal y como ya advirtieron otras investigaciones (Hernández-Mora y Ballester, 2011; Parés, 2011), los usuarios tradicionales del agua (principalmente regantes y usuarios hidroeléctricos) todavía pueden utilizar canales de comunicación paralelos, puertas traseras para influir en los procesos de elaboración de la política de aguas.

Por otro lado, constatamos también que los principales departamentos del gobierno autonómico con competencias en ámbitos relacionados con la gestión del agua (agricultura, industria, turismo o planificación territorial) ni participaron en el proceso ni tomaron en consideración las propuestas surgidas del mismo (con la excepción de los dos departamentos promotores del proceso).

\subsection{Pautas de comportamiento}

En esta sección analizaremos las pautas de comportamiento de los actores en relación al proceso deliberativo, pues creemos que también pueden ser explicativas de las consecuencias de la DMA en nuestro caso de estudio. Nos centraremos en dos tipos de pautas de comportamiento: la conducta de los participantes, por un lado, y el papel desempeñado por las instituciones promotoras del proceso por el otro. En este último caso nos interesa especialmente ver cómo se ha desarrollado el liderazgo político del proceso deliberativo, pues entendemos que es un factor clave para garantizar los impactos del mismo.

En relación a la conducta de los participantes debemos destacar que a lo largó del proceso se fue construyendo un clima de respeto mutuo que facilitó enormemente el trabajo. Sin embargo, este clima debe ser interpretado como un resultado del propio proceso, pues éste no era el punto de partida. El inicio del proceso estuvo caracterizado menos por el respeto y más por la desconfianza (respecto a la administración) y el recelo (respecto a los otros participantes). Las relaciones, por consiguiente, se condujeron con cierta precaución expectante, inicialmente reacia a la colaboración e, incluso, con cierta tendencia a replegarse sobre las propias fuerzas de cada uno de los actores. El agua es un asunto conflictivo y en el que históricamente las oportunidades de interacción entre los diferentes actores han sido escasas, lo que contribuye a explicar estos inicios difíciles. De hecho, quizás el reto más importante del proceso era superar estas fases iniciales, donde sólo se intercambiaban miradas desconfiadas, y avanzar hacia una situación donde, desde la confianza, se intercambiasen palabras constructivas.

El propio diseño del proceso, la tarea de facilitación llevada a cabo por las empresas de consultoría y la buena gestión de las expectativas por parte de los promotores del proceso son tres factores que claramente hicieron posible la transición de un clima de desconfianza y recelo a un acuerdo sobre cómo hacer frente a los desacuerdos, generándose así un nuevo clima de respeto mutuo. Sin embargo, este cambio no se habría producido sin otro hecho clave: la virtud cívica de los participantes. La buenas actitudes de los participantes son indispensables para poder llevar a cabo un proceso de deliberación basada en el respeto mutuo. En nuestro caso de estudio, estas actitudes y valores fueron, en términos generales, excelentes, tal y como demuestran las siguientes citas de distintas personas entrevistadas:

\footnotetext{
"La discusión a veces fue fuerte, pero siempre en positivo" (miembro de una organización ecologista)

"No me lo esperaba (...) una madurez por parte de todos brutal (... ) una normalidad democrática absoluta" (miembro de la ACA)

"Dejando de lado dos o tres ejemplos puntuales, todo transcurrió dentro de unas formas civilizadas" (industrial)
} 
"Hubo mucho feeling entre todos los que estábamos allí (...) La comunicación entre todos mejoró (...) y todo el mundo vio que tanto los unos como los otros teníamos problemas similares." (miembro de una organización ecologista)

Sin minimizar los hechos citados anteriormente, también hay otros elementos que deben mencionarse para entender por qué el proceso de deliberación se desarrolló con este clima de respeto mutuo. Por un lado, la ausencia de algunos actores importantes evitó la aparición de algunas posiciones que hubieran estimulado el conflicto. Por otro lado, el hecho de que algunas cuestiones conflictivas fueron excluidos del debate también facilitó que las relaciones fueran menos tensas. De hecho, los únicos momentos de cierta conflictividad se vivieron cuando se trató un tema concreto (que no poco importante) en el que las posiciones de los actores eran muy contrapuestas: el debate alrededor de los caudales mínimos que debían garantizar el buen estado ecológico de las masas de agua.

"No todo el mundo estaba allí y eso limitó el posible conflicto en el debate" (miembro de la ACA)

"El proceso estaba demasiado orientado a ponerse de acuerdo en las ideas maestras, en una serie de cosas que eran bastante compartidas (...) Se hablaba más en términos generales y algunos temas se escondían" (miembro de la ACA)

Por otra parte, el papel desempeñado por los técnicos de la ACA fue también un hecho clave para explicar la buena marcha de los talleres de deliberación. Casi todos los entrevistados, especialmente los miembros de las organizaciones sociales y ambientales, han destacado la labor realizada por los técnicos: asesoramiento, resolución de dudas, la presentación de documentos, etc.

"El compromiso de los técnicos ha sido muy positivo (...), han trabajado con las mejores intenciones y con absoluta disponibilidad. En muchos de los casos nos han ayudado y han proporcionado información muy valiosa" (miembro de una organización ecologista).

En resumen, pues, vemos que aunque diversos factores facilitaron el clima de respeto mutuo y el trabajo sobre el consenso; el comportamiento de todos y cada uno de los actores participantes fue un elemento de gran valor para que el proceso fuera exitoso.

El lado negativo de los patrones de comportamiento claramente viene en parte determinado por las carencias del liderazgo político del proceso. El proceso de deliberación fue posible gracias a la participación política de los dos departamentos del gobierno catalán que la promovieron: el Departamento de Medio Ambiente y Vivienda, a través de la Agencia Catalana del Agua, y el Departamento de Relaciones Institucionales y Participación, a través de su Dirección General de Participación Ciudadana. Este compromiso político se materializó mediante una re-estructuración administrativa, creando dentro del ACA un órgano administrativo específico para llevar a cabo el proceso, y una importante dotación de recursos económicos y humanos invertidos en el proceso. Al mismo tiempo, sin embargo, hemos identificado una falta de liderazgo político. Los políticos responsables del proceso lo apoyaron claramente, pero su implicación en el proceso era muy limitada: no participaron activamente en los talleres, no explicaron el proceso a los medios de comunicación y no lograron involucrar a otros departamentos clave del gobierno autonómico (agricultura, política territorial, industria, turismo...). En otras palabras, no lideraron suficientemente el proceso como para situarlo entre las prioridades políticas del gobierno catalán. El proceso se hizo pero no fue incorporado en la agenda política del gobierno.

Como consecuencia de ello se derivaron las siguientes debilidades del proceso deliberativo. En primer lugar, algunos temas quedaron fuera del debate. Tal vez un fuerte liderazgo político podría haber forzado la incorporación de los temas más conflictivos en el debate. En segundo lugar, la ya mencionada ausencia significativa de algunos actores se podría deber a que algunos actores habrían percibido que el gobierno no le daba suficiente importancia a esta cuestión. Con un liderazgo político más fuerte quizá se habría logrado también una mayor participación de algunos de estos actores. En tercer lugar, la falta de transversalidad derivada de la nula implicación de algunos departamentos clave, como los que tienen competencias en la agricultura, industria, turismo y política territorial. Por último, las limitaciones de la estrategia comunicativa que hemos explicado más arriba también podrían haberse resuelto si el gobierno hubiera liderado mejor el proceso. Los gobiernos suelen tener un fácil acceso a los medios de comunicación. El caso del gobierno catalán no es una excepción en este sentido, especialmente en 
relación a los medios de comunicación autonómicos y locales. Así, los déficits de comunicación que hemos identificado en el proceso se podrían haber resuelto con un mayor esfuerzo, por parte del gobierno, para difundirlo a través de los medios de comunicación autonómicos y locales, cosa que también habría contribuida a que el proceso hubiera llegado al público en general, más allá de los actores interesados a los que el proceso sí que logró llegar. En síntesis, podríamos decir que en el proceso de deliberación analizado hubo un gran compromiso político por parte de los departamentos del gobierno promotores del mismo pero, al mismo tiempo, no hubo en absoluto un liderazgo político fuerte y claro por parte del conjunto del gobierno catalán.

\section{LAS CONSECUENCIAS DE LA DELIBERACIÓN}

Una vez analizadas las características procedimentales de nuestro estudio de caso, veamos en qué medida estas han determinado los resultados de la deliberación. Nos fijaremos en dos tipos de consecuencias de la deliberación: los resultados tangibles y los resultados intangibles.

\subsection{Resultados tangibles}

El primer elemento que debe destacarse es que el proceso de deliberación dio lugar a unas conclusiones claras y tangibles que, una vez validadas por los participantes, se exhibieron públicamente en el sitio web de la ACA. El resultado fueron 1.529 propuestas de diversa índole a las que el Departamento de Medio Ambiente y Vivienda del gobierno catalán dio la respuesta que se muestra en la tabla 2.

Tabla 2. Resultados del proceso

\begin{tabular}{|l|l|l|}
\hline \multicolumn{1}{|c|}{ Propuestas } & Número & \multicolumn{1}{c|}{$\%$} \\
\hline Aceptadas pero ya planificadas & 608 & $40 \%$ \\
Aceptadas e innovadoras & 356 & $23 \%$ \\
Rechazadas & 51 & $3 \%$ \\
Transferidas a otros Departamentos & 514 & $34 \%$ \\
Total & 1.529 & $100 \%$ \\
\hline
\end{tabular}

Fuente: Agència Catalana de l'Aigua, 2010

Así, un total de 964 propuestas (63\% del total de propuestas realizadas) fueron incorporadas en el plan de cuenca aprobado por el gobierno catalán. Una gran mayoría de estas propuestas, sin embargo (608), ya habían sido previstas por los técnicos redactores del plan con anterioridad a la celebración del proceso deliberativo. Las 356 propuestas restantes son las que se incorporaron al plan y significaron una innovación, una mejora, respeto al plan que habrían hecho los técnicos del ACA si no se hubiera llevado a cabo el proceso deliberativo. Desde este punto de vista, podemos decir que el proceso de deliberación tuvo un impacto significativo en la política de gestión de las cuencas hidrográficas, aunque el potencial innovador de la deliberación fue más bien limitado.

Sin embargo, esta no es la percepción que la mayoría de los participantes tiene en relación a los resultados del proceso deliberativo. Las personas entrevistadas del sector agrícola e industrial se mostraron muy escépticas sobre el efecto real que el proceso deliberativo había tenido, básicamente porque consideran que sus intereses no están suficientemente incluidos en el plan final. Las organizaciones ambientalistas, por otro lado, muestran una mayor diversidad de opiniones. Algunas de ellas evalúan positivamente la incorporación de sus propuestas en el plan definitivo, mientras que otras se muestran muy escépticas.

Observamos que cuando preguntábamos a los entrevistados sobre temas concretos (y tal vez menos importantes), su opinión en relación al impacto sustantivo de la deliberación era más positiva, reconociendo que en aquella cuestión concreta el proceso deliberativo sí que había logrado incidir. Por el contrario, cuando se les pregunta acerca de temas conflictivos o más estratégicos (como los que afectan a los caudales, la agricultura, la energía o el modelo urbanístico), entonces su percepción acerca de la influencia del proceso era más negativa. La falta de liderazgo político descrita anteriormente y la nula participación de los departamentos son dos factores muy relevantes para poder comprender esta situa- 
ción. La falta de liderazgo ha condicionado que algunos temas de carácter estratégico fueran abordados de forma deliberativa, mientras que la nula participación de algunos departamentos explica que las cuestiones relativas a estos departamentos fueran apartadas del debate. En consecuencia, el proceso no permitió que se llevara a cabo un debate articulado y propositivo sobre cuál debería ser, por ejemplo, el modelo urbanístico, el modelo agrícola, el modelo energético o el modelo turístico que permitiera lograr el buen estado ecológico de las masas de aguas para el año 2015. Por el contrario, el proceso se limitó a discutir y proponer medidas concretas del ámbito competencial del Departamento de Medio Ambiente y Vivienda.

Por último, otro resultado tangible del proceso deliberativo tiene que ver con la estructura de la administración del agua en Cataluña. Como consecuencia del proceso deliberativo la Agencia Catalana del Agua modificó su organización interna. Se creó una nueva unidad de la participación dentro de la agencia y estaba previsto que se crearan nuevos consejos de cuenca a partir de las distintas unidades participativas con las que se llevó a cabo el proceso deliberativo. Aunque estas decisiones quedaron alteradas por el cambio de gobierno que se produjo en Cataluña a finales del año 2010, la realidad es que el proceso deliberativo ha abierto un nuevo marco de relaciones entre la administración y la sociedad. De tal forma que, actualmente, la cantidad y la diversidad de actores relacionados con el agua que deben ser tenidos en cuenta por parte de la administración en la gestión de agua son, indudablemente, muy superiores a los de la etapa precedente.

\subsection{Resultados intangibles}

Además de los resultados tangibles que acabamos de describir, el proceso dio lugar también a otro tipo de efectos de una naturaleza más intangible. Podemos identificar cuatro tipos de resultados intangibles en el caso que hemos estudiado: la legitimidad política, el logro de una posición de interés público, el reconocimiento mutuo entre los interesados y el aprendizaje social producido por el proceso. Veámoslos con un poco más de detalle.

Debido a la alta calidad deliberativa del proceso llevado a cabo en Cataluña, la legitimidad de la política de gestión de cuencas hidrográficas se ha fortalecido. En términos generales, todas las partes interesadas reconocen que el proceso de deliberación ha sido bien desarrollado y, como resultado, su legitimidad ha aumentado. Como hemos visto, sin embargo, incluso si los participantes están satisfechos con el proceso no están satisfechos con los resultados sustantivos. Obviamente, la insatisfacción con los resultados reduce la legitimidad de la decisión final adoptada por el gobierno autonómico. Una vez más, estas percepciones deben ser matizadas en función del perfil de los participantes. Mientras que los actores institucionales están satisfechos con el proceso y los resultados, los actores agro-ganaderos y forestales tienden a estar satisfechos con el proceso, pero muy satisfechos con los resultados. En el centro, las organizaciones ambientalistas están más satisfechos con el proceso y menos con los resultados y los agentes económicos están decepcionados con los resultados, pero entienden que, al igual que en una negociación, el resultado final conlleva simultáneamente pérdidas y ganancias desde el punto de vista de sus intereses particulares.

Esta insatisfacción con el proceso, como se ha sugerido anteriormente, puede ser consecuencia de una importante falta de cultura deliberativa, pues observamos que todos los actores entrevistados miden su satisfacción o insatisfacción con los resultados en función de qué parte de sus intereses particulares han logrado incorporar en este resultado. Desde otra perspectiva, en cambio, podríamos interpretar que esta insatisfacción generalizada podría ser un indicador de que se ha logrado un equilibrio entre los intereses; un hecho que también se vería corroborado por el escaso número de propuestas rechazadas y por el alto grado de consenso alcanzado durante el proceso. En este sentido, y este es el segundo resultado intangible que queremos destacar, el proceso de deliberación habría logrado pasar de una suma de intereses particulares contradictorios a la construcción de un una posición de interés público coherente y consensuada.

El tercer resultado intangible del proceso ha sido la mejora de las relaciones entre las partes interesadas. Sin ningún tipo de duda, el clima de respeto mutuo generado durante y como consecuencia del proceso tiene una relación causa-efecto. Todos los actores, pero en especial las organizaciones ambientalistas, dan un valor muy positivo a las nuevas relaciones que se han establecido a través del proceso porque 
todos ellos comparten un tema específico: la gestión del agua. Todo el mundo ha reconocido a "los otros" como actores importantes en la gestión del agua y la mayoría han sacado provecho de estas nuevas relaciones. Las organizaciones ambientalistas y las comunidades agrícolas han compartido argumentos y han establecido algunas alianzas, los agentes económicos han descubierto otros puntos de vista sobre la gestión del agua y la ACA ha formalizado una red de actores que deben tenerse en cuenta en el diseño e implementación de cualquier política de aguas.

Por último, en relación con el aprendizaje social, vemos que más allá de la cantidad de propuestas innovadoras (tabla 2), la gran mayoría de los participantes creen que el alcance de esta innovación ha sido muy limitado. Se ha innovado y eso ha dado lugar a algunos nuevos aprendizajes pero, para decirlo de una manera muy simple, han sido pequeños aprendizajes porque las aportaciones sustantivas derivadas del proceso fueron poco significativas. De hecho, incluso las instituciones que promovieron el proceso reconocen que los efectos intangibles producidos por el propio proceso (complicidades, reconocimiento mutuo, etc. ) son más importantes que las contribuciones sustantivas.

\section{CONCLUSIONES}

En este artículo hemos evaluado un proceso deliberativo ejemplar desarrollado bajo el paraguas de la Directiva Marco del Agua. Los resultados de nuestra evaluación demuestran que, efectivamente, el proceso cumple las características principales de la democracia deliberativa sugeridas por Gutmann y Thompson (2004): el proceso ha conducido a una decisión adoptada desde el intercambio de argumentos y la voluntad de integración, el intercambio de argumentos ha sido comprensible y accesible, las decisiones que resultaron del proceso han sido eficaces y, finalmente, la deliberación ha sido pautada economizando los desacuerdos morales. Sin embargo, nuestra evaluación también muestra que la gran mayoría de los participantes son muy críticos con los resultados del proceso. Para resumir, podemos afirmar que los participantes están satisfechos con el proceso pero insatisfechos con sus resultados.

Concluimos, pues, que la realización de un buen proceso deliberativo es una condición necesaria pero no suficiente para lograr mejorar los resultados de la política de aguas. Si se quiere que la deliberación influya realmente en la política de aguas, es necesario que esta venga acompañada de un liderazgo político claro y comprometido con el proceso. Además, también resulta del todo indispensable que la forma de abordar el problema (en este caso la gestión de cuencas hidrográficas) se ajuste a la realidad y la complexidad del mismo. En otras palabras, la gestión de cuencas hidrográficas es una cuestión compleja que requiere de un enfoque integral. Abordar esta cuestión desde una perspectiva estrictamente sectorial (medioambiental en este caso) no sólo limita las posibilidades para lograr una resolución satisfactoria de una problemática que tiene causas y consecuencias multisectoriales y multiescalares sino que también entra en contradicción con la visión de los participantes. Estos, por lo menos en el caso estudiado, han demostrado tener una visión mucho más holística de la problemática que la propia administración pública, que sigue actuando a partir de una rígida fragmentación departamental.

Al mismo tiempo, hemos visto también como algunas de las debilidades del proceso han condicionado las consecuencias (los efectos) del mismo. Así, los futuros procesos deliberativos sobre la gestión de cuencas hidrográficas deberían tener en cuenta algunos de los elementos procedimentales analizados para mejorar no sólo sus resultados, sino también la percepción de estos resultados. En este sentido, cabe destacar al menos tres sugerencias. En primer lugar, la planificación y la gestión de cuencas hidrográficas debe abordarse de manera integral, incluyendo todas las dimensiones y áreas. Eso significa que ninguna cuestión debería ser excluida del debate y que, a través de un modelo de gobernanza en red, deben participar en el proceso todos los departamentos del gobierno que estén en relación con la política de aguas. En segundo lugar, es preciso mejorar la estrategia de comunicación, no sólo para enfrentar una vasta diversidad de participantes sino también para evitar la frustración de las expectativas de los ciudadanos. Y por último, pero no menos importante, debe realizarse un cambio cultural para aprender e interiorizar la gestión deliberativa de los problemas colectivos. Los procesos deliberativos sólo serán capaces de tener éxito cuando los políticos, la administración pública, las partes interesadas y la sociedad en su conjunto adopten una cultura política deliberativa. 


\section{REFERENCIAS BIBLIOGRÁFICAS}

ANTUNES, P.; KALLIS, G.; VIDEIRA, N.; SANTOS, R. (2009): "Participation and evaluation for sustainable river basin governance" en Ecological Economics, no 68, pp. 931-939.

ARNSTEIN, S. (1969): "A ladder of citizen participation" en Journal of the American Institute of Planners, vol. $35, n^{\circ} 4$, pp. 216-224.

BAKER, M.; STEPHEN, H.; SHERRIFF, G. (2010): "Getting involved in plan making: participation and stakeholder involvement in local and regional spatial strategies in England" en Environment and Planning C: Government and Policy, vol. 28, n 4, pp. 574-594.

BLANCO, I.; BALLESTER, M. (2011): “¿Participar para transformar? La experiencia de los Presupuestos Participativos en la provincia de Barcelona” en Gestión y Análisis de Políticas Públicas, nº 5, pp. 117-144.

BLANCO, I.; LOWNDES, V. (2011): "Does community participation improve the quality of policymaking? Exploring and explaining the effects of democratic innovations in neighbourhood regeneration" en Joint Sessions of Workshops of the ECPR.

BOHMAN, J.; REHG, W. (1997): Deliberative Democracy. The MIT Press. London.

BOBBIO, L. (2010): "Types of deliberation" en Journal of Public Deliberation, vol. 6, nº 2, pp. 1-24.

BRUGUÉ, Q. (2004): "Modernizar la administración desde la izquierda: burocracia, nueva gestión pública y administración deliberativa" en Revista del CLAD. Reforma y Democracia, no 29, pp. 1-16.

BRUGUÉ, Q.; PARÉS, M. (2012): "Entre la deliberación y la negociación: el caso de la Mesa de la Montaña en Aragón" en Revista de Estudios Políticos, n 158, pp. 55-101.

CONRAD, E.; CASSAR, L.F; CHRISTIE, M.; FAZEY, I. (2011): "Hearing but not listening? A participatory assessment of public participation in planning" en Environment and Planning C: Government and Policy, vol. 29, nº 5, pp. 761-782.

DE STEFANO, L. (2010): "Facing the water framework directive challenges: a baseline of stakeholder participation in the European Union" en Journal of Environmental Management, nº 91, pp. 1332-1340.

ESPLUGA, J.; BALLESTER, A.; HERNÁNDEZ-MORA, N.; SUBIRATS, J. (2011): "Participación Pública e inercia institucional en la gestión del agua en España” en Revista Española de Investigaciones Sociológicas, $\mathrm{n}^{\circ} 134$, pp. 3-26.

FONT, J.; BLANCO, I. (2006): Polis, la ciudad participativa. Diputació de Barcelona. Barcelona.

FUNG, A. (2003): "Survey Article: Recipes for Public Spheres: Eight institutional design choices and their consequences" en The Journal of Political Philosophy, vol. 11, n 3, pp. 338-367.

FUNG, A. (2006): "Varieties of participation in complex governance" en Public Administration Review, $\mathrm{n}^{\circ} 66$, pp. 66-75.

GROVES, C.; MUNDAY, M.; YAKOVLEVA, N. (2013): "Fighting the pipe: neoliberal governance and barriers to effective community participation in energy infrastructure planning" en Environment and Planning C: Government and Policy, vol. 31, n 2, pp. 340-356.

GUTMANN, A.; THOMPSON, D. (2004): Why deliberative democracy. Princeton University Press. Princeton.

HAUS, M.; HEINELT, H.; STEWART, M. (2005): "Introduction" en Urban governance and democracy: Leadership and community involvement. Haus, M.; Heinelt, H.; Stewart, M. (eds). Routledge. London.

HENDRIKS, C. (2006): "When de Forum Meets Interest Politics: Strategic Uses of Public Deliberation" en Politics \& Society, vol. 34, no 4 , pp. 571-602.

HENDRIKS, C.; DRYZEK, J.; HUNOLD, C. (2007): "Turning Up the Heat: Partisanship in Deliberative Innovation” en Political Studies, nº 55, pp. 362-383. 
HERNÁNDEZ-MORA, N.; BALLESTER, A. (2011): "Public participation and the role of social networks in the implementation of the water framework directive in Spain" en Ambientalia. Revista Interdisciplinar de las Ciencias Ambientales, pp. 1-21.

HUITEMA, D.; MEIJERINK, S. (2010): "Realizing water transitions: the role of policy entrepreneurs in water policy change" en Ecology and Society, vol. 15, n 2, pp. 26.

KAIKA, M. (2003): "The Water Framework Directive: A New Directive for a Changing Social, Political and Economic European Framework" en European Planning Studies, vol. 11, n 3, pp. 299-316.

KALLIS, G. (2006): "Participatory methods for water resource planning" en Environment and Planning C: Government and Policy, $\mathrm{n}^{\circ} 24$, pp. 215-234.

KALLIS, G.; BUTLER, D. (2001): "The EU water Framework Directive: measures and implications" en Water Policy, no 3, pp. 125-142.

LA CALLE, A. (2009): "La participación pública active y real en la política del agua: necesidad y deber" en Anduli, Revista Andaluza de Ciencias Sociales, nº 8, pp. 67-82.

LANZ, K.; SCHEUER, S. (2001): EEB Handbook on EU Water Policy under the Water Framework Directive. EEB. Brussels.

LO, A.Y.; ALEXANDER, K.S.; PROCTOR, W.; RYAN, A. (2013): "Reciprocity as deliberative capacity: lessons from a citizen's deliberation on carbon pricing mechanisms in Australia" en Environment and Planning C: Government and Policy, vol. 31, nº 3, pp. 444-459.

MOSTERT, E. (2003): “The challenge of public participation” en Water Policy, vol. 5, n 2, pp. 179-197.

NEWIG, J.; PAHL-WOSTL, C.; SIGEL, K. (2005): “The role of public participation in managing uncertainty in the implementation of the Water Framework Directive" en Environmental Policy and Governance, vol. $15, n^{\circ} 6$, pp. 333-343.

NEWIG, J.; GÜNTHER, D.; PAHL-WOSTL, C. (2010): "Synapses in the Network: Learning in Governance Networks in the Context of Environmental Management" en Ecology and Society, vol. 15, n 4, 24 p.

PAPADOPOULUS, Y.; WARIN, P. (2007): “Are Innovative, Participatory and Deliberative Procedures in Policy Making Democratic and Effective?" en European Journal of Political Research, n 46, pp. 445-472.

PARÉS, M. (2009): Participación y calidad democrática. Evaluando las nuevas formas de democracia participativa. Ariel. Barcelona.

PARÉS, M. (2011): "River basin management planning with participation in Europe: from contested hydro-politics to governance-beyond-the-state" en European Planning Studies, vol. 19, n 3, pp. 457-478.

RUDDIN, L. (2006): "You can generalize stupid! Social scientists, Bent Flyvbjerg, and Case Study Methodology" en Qualitative Inquiry, n 12, pp. 797-812.

SABEL, C.; ZEITLIN, J. (2008): "Learning from difference: the new architecture of experimentalist governance in the EU" en European Law Journal, vol. 14, n 3, pp. 271-327.

SCHKADE, C.; SUNSTAIN, C.; HASTIE, R. (2006): "What Happened on Deliberation Day?" en Chicago John M. Olin Law E Economics Research Series Working Paper 298, The University of Chicago.

SUBIRATS, J. (2003): Elementos de nueva política. Centro de Cultura Contemporánea de Barcelona. Barcelona.

SUNSTAIN, C.; HASTIE, R. (2008): "Four Failures of Deliberating Groups" en Public Law and Legal Theory Working Paper 215, The University of Chicago.

WAGENAAR, H. (2007): "Governance, complexity and democratic participation: how citizens and public officials harness the complexities of neighbourhood decline 2 en The American Review of Public Administration, $\mathrm{n}^{\mathrm{o}} 37$, pp. 17-50.

YIN, R.K. (2003): Case Study Research. Design and methods. Sage. London. 